\title{
MODAL TESTS AND ANALYSIS OF A RADIAL IMPELLER AT REST: INFLUENCE OF SURROUNDING AIR ON DAMPING
}

\author{
C. Gibert ${ }^{a *}$ L. Blanc ${ }^{a}$, P. Almeida ${ }^{a}$, X. Leblanc ${ }^{a}$, J.-P. Ousty ${ }^{b}$, F. Thouverez ${ }^{a}$, J.-P. Laîné ${ }^{a}$ \\ (a) École Centrale de Lyon, Laboratoire de Tribologie et Dynamique des Systèmes \\ 36 avenue Guy de Collongue, 69134 Ecully Cedex, France \\ (b) Turbomeca - Safran group, 64511 Bordes cedex, France \\ claude.giberteec-lyon.fr
}

\begin{abstract}
$H C F$ risk assessment for turbomachinery blades requires the prediction of vibratory levels, which in turn requires fine damping quantification. This issue is especially sensitive for structures with low structural damping such as monobloc centrifugal compressor disks (blisks). The material composing blisks and aerodynamic flow both contribute to damping phenomena. A strategy for non-aerodynamic damping characterization is to perform experiments in vacuum.

This paper focuses on the use of modal tests in vacuum to estimate material damping under non-rotating conditions. Experiments are performed on an isolated impeller manufactured from a single piece in a vacuum chamber at different air pressure levels ranging from 10 mbar to 1 bar. Strong dependency of damping ratios on pressure can be found on the first flexural mode, leading to two types of application.

Firstly, measurements enable assessing the validity of extrapolations of non-aerodynamic damping from measurements sometimes performed under less thorough vacuum conditions. Basic fluid-structure interaction models are used to interpret and quantify the evolution of modal quantities when air is progressively removed. Secondly, vacuum measurements can give frequency response functions ( $F R F S$ ) with much greater separation between resonance peaks. In this study, the damping ratio found in vacuum condition are $3 \%$ of these at ambient pressure corresponding to a magnitude $30 \mathrm{~dB}$ higher at resonance peaks. This contrasts with in-air measurements on cyclic symmetry struc-
\end{abstract}

\footnotetext{
*Address all correspondence to this author.
}

tures, like blisks, with high modal density that make the direct interpretation of FRFs and their modal analysis more difficult.

\section{NOMENCLATURE}

- norm normalized quantity

$\eta$ loss factor

$v$ Poisson coefficient

$\omega, \omega_{k}, \Delta \omega$ excitation frequency and resolution (in $\mathrm{rad} / \mathrm{s}$ )

$\omega_{0}$ natural frequency of conservative system (in $\mathrm{rad} / \mathrm{s}$ )

$\omega_{0}^{\prime}$ natural frequency in air (in $\mathrm{rad} / \mathrm{s}$ )

$\omega_{1}$ first flexural mode natural frequency

$\rho_{0} \quad$ air density

$\rho_{m}$ material density

$\zeta$ critical damping ratio

$\zeta_{0}$ critical damping ratio in vacuum

$a, b, h$ length, width and thickness of rectangular plate

$c$ velocity of sound in the air

$c_{m} \quad$ velocity of sound in the material

$k$ parameter characteristic of air-structure coupling type

$p^{+}, p^{-} \quad$ instant air pressures

$w$ displacement

$C_{c r} \quad$ critical damping

$D$ total energy dissipated during one cycle

E Young modulus

$F_{p}$ resultant pressure force

$M, C, K$ mass, structural viscous damping, stiffness

$M_{a}, C_{a}$ added mass, added damping

$P \quad$ static air pressure 
$U$ maximum strain energy during one cycle

$Z_{r}=\left(R_{r}+j X_{r}\right) \quad$ radiation impedance

DSP Digital Signal Processing

FRF Frequency Response Function

$N D$ Nodal Diameter

ODS Operational Deflection Shape

PMMA Polymethyl Methacrylate

PZT Lead Zirconate Titanate piezoelectric material

SDOF Single Degree Of Freedom

SLDV Scanning Laser Doppler Vibrometry System

\section{INTRODUCTION}

Turbomachinery blade vibration damping results from several energy dissipation phenomena. Three energy transfer paths can be distinguished for the most general cases of mechanical systems immersed in a fluid [1]: "fluid damping due to fluid drag, viscous dissipation and radiation to the surrounding fluid"; "internal material damping due to yielding, heating ... and internal energy dissipation of materials"; "structural damping due to friction, impact, scraping and the motion of fluid trapped (squeeze film damping, etc.) in a joint".

Designing monobloc centrifugal impellers requires assessment for both the aerodynamic damping provided by the flow during operation and for material damping. Indeed, no structural damping occurs in such structures due to monobloc manufacturing and to the absence of any specific devices such as underplatform dampers, friction rings [2], viscous material coatings, etc. Under such conditions, material damping assessment becomes all the more important as material damping - even low - is the only source of dissipation when aerodynamic damping becomes null or even negative, with the risk of instabilities. In addition to flutter margin prediction, material damping data is also required to calculate finely forced vibration levels when assessing HCF risk. In this study, we focus on material damping assessment.

Material damping refers to energy dissipation when a volume of the material is subjected to cyclic stresses. Many complex mechanisms are involved "associated with internal reconstruction such as molecular dislocations and stress changes at grain boundaries" [3]. Analysis is difficult due to non-linearity and variability inside the material. Nevertheless, indicative values of losses are obtained by experimental measurements performed on samples of specific materials. Influence parameters are a priori the composition and temperature of the material and stress distribution, which depends on the geometry and on the kind of loading mechanism, especially in terms of frequency and stress level [4]. The loss factor, $\eta$, is used as the measurement of material damping for a given part. It is defined as the ratio of total energy dissipated $D$ over the maximum strain energy $U$ during one cycle of vibration:

$$
\eta=\frac{D}{2 \pi U}
$$

$\eta$ is subjected to estimates varying from $0.00104 \%$ to $1.7 \%$ for a twisted titanium alloy plate as a function of the mode tested, the stress level and the presence or absence of air; in vacuum, the range of values ranged from $0.00104 \%$ to $0.051 \%$ [5]. Such disparities have been observed in the literature and in studies of axial compressor blisks in unpublished works. Compared to axial configurations, radial compressor blisks present very different geometries and vibrate according to specific mode shapes, making it difficult to draw accurate analogies with axial machine material damping levels. Thus it is necessary to carry out a specific study.

A fair number of papers have dealt with this issue in the open literature. In [6], non-aerodynamic damping was measured under vacuum conditions and substracted from overall damping obtained in rotating facilities to estimate aerodynamic damping. The experimental approach was based on [7] relating to piezoelectric excitation. In [8, 9], vacuum tests were performed on an aluminum impeller mounted on a shaft subjected to piezo-excitation and instrumented with strain gages and pressure transducers on the surface of the blades. The results focused on the first two eigenmodes of a main blade. Good agreement was obtained with the material damping extrapolated from measurements performed under aerodynamic excitation for decreasing inlet pressure values. In [10], with a specific engine representative of operating conditions, analogous tests revealed that aerodynamic damping could be about 3 times higher than material damping, then the latter is far from negligible.

Here, the purpose is to present and discuss experimental results obtained under vacuum conditions in view to assessing material damping under the "purest" possible experimental conditions. Contrary to other works constrained by the need to perform rotating or in-flow measurements in addition to structural measurements, here the setup is dedicated to removing the influence of boundary conditions and embarked instrumentation. To achieve this: 1/ the impeller is supported punctually instead of being clamped, to prevent energy from being lost in the mounting; $2 /$ the instrusivity of the measurement device is minimized as a laser Doppler vibrometer (LDV) is used.

In the first part of this paper, the principle of vibratory measurements in vacuum is recalled to provide a theoretical model of the influence of still air pressure on modal parameters. Then the experimental setup and procedure are described. Lastly, the results are presented and discussed in terms of structural damping quantification and also in terms of modal parameter identification, since lower damping in vacuum leads to the clear separation of FRF peaks and is useful for modal analysis. It is shown that 
levels at resonance of 1 st flexural modes while testing at 10 mbars are $30 \mathrm{~dB}$ higher, with damping ratios 30 times lower, compared to results obtained at ambient pressure.

\section{PRINCIPLE OF VIBRATORY MEASUREMENTS IN VACUUM}

\subsection{Hypotheses on fluid - structure interaction}

The aim is to develop a phenomenological model as the basis for formulating a measurement protocol. Let us consider the fluid-structure system composed of the impeller and the surrounding still air. The non-conservative effects associated with the fluid can be linked with the radiation of acoustic waves at "infinity", with viscosity effects and with turbulence if the amplitude of the blades is high enough. Here, air viscosity and turbulent losses are neglected compared to radiation losses. Thus air is modeled as a perfect gas that obeys linear acoustics laws. The impeller is assumed to be weakly damped in vacuum and able to freely oscillate. Under such hypotheses, it is conceivable to provide a modal description of the dynamical behaviour of the system. It is also assumed that intermodal coupling is negligible. In the vicinity of a natural frequency determined in vacuum, the system is therefore analogous with a classical SDOF oscillator.

\subsection{Basic phenomena}

A qualitative model is used in view to determining how vacuum affects modal quantities. It is taken from [11-13]. The system is a rigid baffled flat piston of mass $M$, surface $S$, hanging from a spring of stiffness $K$ and fitted with a viscous damper of coefficient $C$ (see Fig. 10. It is placed in an infinite plane rigid baffle separating two half-spaces. Let $w$ be the piston's displacement along the axis perpendicular to the baffle. When the mass is excited by a sinusoidal force $F_{0} e^{j \omega t}$, the equilibrium equation is:

$$
\left(M+M_{a}\right) \ddot{w}+\left(C+C_{a}\right) \dot{w}+K w=F_{0} e^{j \omega t} .
$$

Terms $M_{a}$ and $C_{a}$ contain the contribution of air pressure force $F_{p}$. Let $p^{+}$and $p^{-}=-p^{+}$designate the fluid pressure on the piston faces. $F_{p}$ and piston speed $\dot{w}$ are linked by radiation impedance $Z_{r}$ by:

$$
-\frac{F_{p}}{S}=\frac{2}{S} \iint_{S} p^{+} d S=Z_{r} \dot{w}=\left(R_{r}+j X_{r}\right) \dot{w} .
$$

Then, $M_{a}=S X_{r} / \omega$, with $X_{r}$ the reactive part of $Z_{r}$, has an added mass effect and modifies the natural frequency. $C_{a}=S R_{r}$, along with $R_{r}$, which is the resistive part of $Z_{r}$, adds damping to structural damping. $M_{a}$ and $C_{a}$ are functions of $\omega$. Both terms can modify the structural response and result in measurements that

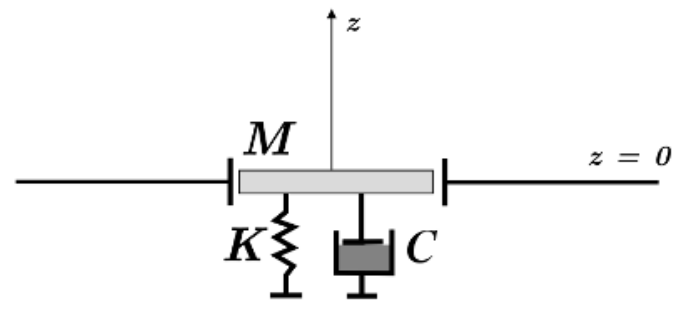

FIGURE 1: Baffled piston SDOF system, adapted from [12]

would give erroneous natural frequencies and material damping if modal data identified in ambient air were assimilated with those expected in vacuum.

Here we presume - later confirmed by the experiments - that the influence of coupling with air is weak. Thus the complex natural frequency of the system in air is derived from the natural frequency in vacuum through a first order perturbation analysis. Moreover, it is shown that for linear acoustics, the radiated pressure generated by each point of the structure is proportional to the characteristic impedance of fluid $\rho_{0} c$ with $\rho_{0}$ being the density and $c$ the speed of the acoustic waves. For a perfect gas, $\rho_{0}$ is proportional to $P$ and $c$ depends only on the temperature. Then $Z_{r}$ is proportional to $P$ and both natural frequency $\omega_{0}^{\prime}$ and critical damping ratio $\zeta$ in air are affine functions of static air pressure $P$. Their values are deduced from $\omega_{0}$ - natural frequency without air or damping - and from $\zeta_{0}$ - critical damping ratio of the system in vacuum by:

$$
\zeta=\zeta_{0}+\alpha\left(\omega_{0}\right) P \quad \text { and } \quad \omega_{0}^{\prime}=\omega_{0}+\beta\left(\omega_{0}\right) P
$$

with $\alpha$ and $\beta$ coefficients depending on $S, M$ and $Z_{r}\left(\omega_{0}\right)$. For the baffled piston (and also for the unbaffled piston). It can be shown analytically that an increase of air pressure is expected to lead to increased damping and a decrease in natural frequency compared to vacuum values.

\subsection{Experimental quantification of material damping}

Consequently, the general principle of the measurement is as follows [12]. Firstly, the vibratory signal is measured for decreasing values of pressure $P$ in a vacuum chamber. Secondly, for each eigenmode considered, natural frequency $\omega_{0}^{\prime}(P)$ and critical damping ratio $\zeta(P)$ are estimated. The slopes of the straight lines fitting the best - in terms of least mean squares - $\omega_{0}^{\prime}(P)$ and $\zeta(P)$ are estimated and their values for a null pressure are extrapolated. $\zeta_{0}=\zeta(P=0)$ stands for structural damping, i.e. material damping under the conditions of the experiment described below. 


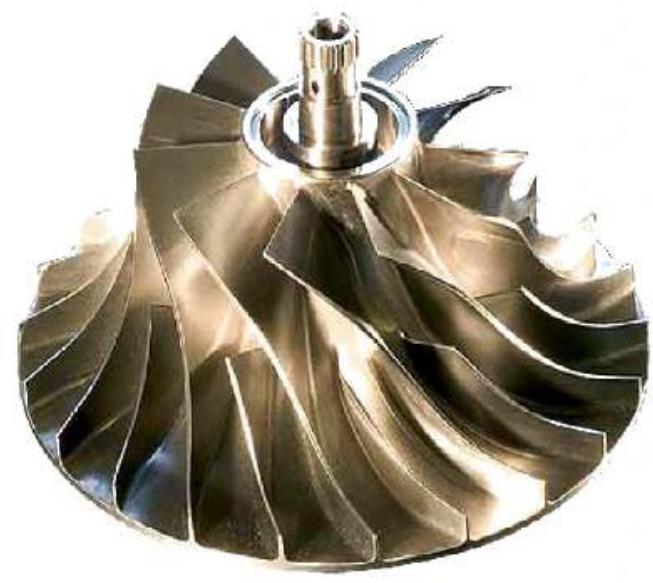

FIGURE 2: Typical centrifugal compressor wheel, courtesy of Turbomeca

\section{EXPERIMENTAL SETUP AND PROCEDURE}

\subsection{Test specimen}

The impeller tested was a centrifugal compressor wheel analogous to that shown in Fig. 2. Its shaft was removed to concentrate on the blading so that it consisted of a single part with ten main blades and ten intermediate blades (or splitter blades). It was made of titanium alloy.

\subsection{Free boundary conditions}

In order to avoid any disturbances from clamping mounts, the impeller was supported close to its center of mass, as shown in Fig. 3. The impeller is simply supported at the end of a rigid cylindrical steel beam. This latter is clamped at its other end. A piece of rubber represented by a black rectangle in the sketch in Fig. 3 is placed between the beam and the impeller to provide a soft dynamic isolation between these two parts. The rubber dimensions are about $10 \times 10 \times 4 \mathrm{~mm}$. It is in contact with the inside of the cylindrical bore of the test piece. The main contact force applied to the impeller is the reaction of the rubber to its weight. Dynamic reaction forces are limited thanks to the very low stiffness of the rubber part. Also, the theoretical modal displacements of the free-free impeller at the bore is minimum and thus minimizing any effect of the support. This is illustrated in Fig. 4 which shows mode shapes obtained through pre-test FE modal calculations for one of the modes of interest. A closer insight at the bore surface would show that the modal displacements are less than 0.02 (compared to 10 on the backside and 300 on the balde).

The modes of the clamped-free beam supporting the impeller on the rubber part are outside the frequency range containing the first ten frequencies of the impeller.

This mounting method also had the advantage of being very

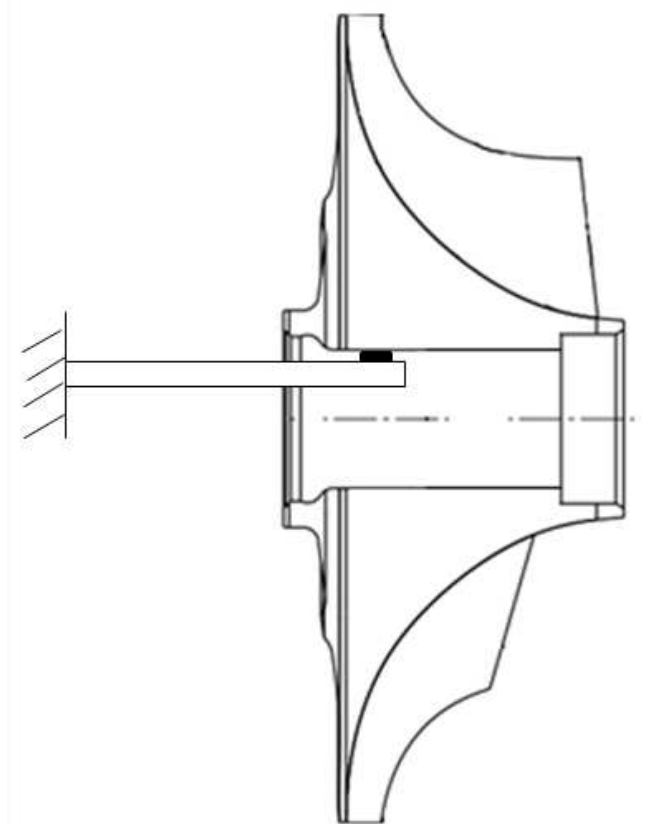

FIGURE 3: Principle of impeller's free mount

simple, well adapted to the specific geometry of the impeller and avoided possible obstruction of the optical path to the measurement points by any elastic strings usually used. In Fig. 4 one can compare the modal displacements both on the blades and on the back side of the impeller.

\subsection{Excitation setup and measurement}

Excitation was provided by a piezoelectric (PZT) translator stack P-802.00 [14] of dimensions $6 \times 6 \times 9 \mathrm{~mm}$, associated with a seismic mass (Fig. 5). The shaker was bonded to the upper side of a piezoelectric force sensor which in turn was attached to the structure. The elongation of the translator, essentially proportional to the tension provided by a piezoelectric driver amplifier, generated inertial reaction forces proportional to the square of the frequencies transmitted to the impeller. The translator was driven with a direct component of $+50 \mathrm{Vdc}$ superimposed with an alternating component (range $+/-50 \mathrm{Vac}$ ) in order to operate within the prescribed 0-100 Volt range of the translator [15].

The input point chosen was in a rather stiff and flat region on the structure but produced a sufficient response level at the measurement points when excited by the shaker (see Fig. 6 at midrange between the impeller center and outer radius). Also, the mass-loading induced by the force sensor (total mass 22.7 $\mathrm{gm}$ ) was slight at this point. It was evaluated using a first order approximation of the sensitivity of natural frequencies w.r.t. a local punctual mass, and was found to be less than $\Delta \omega$ the fre- 

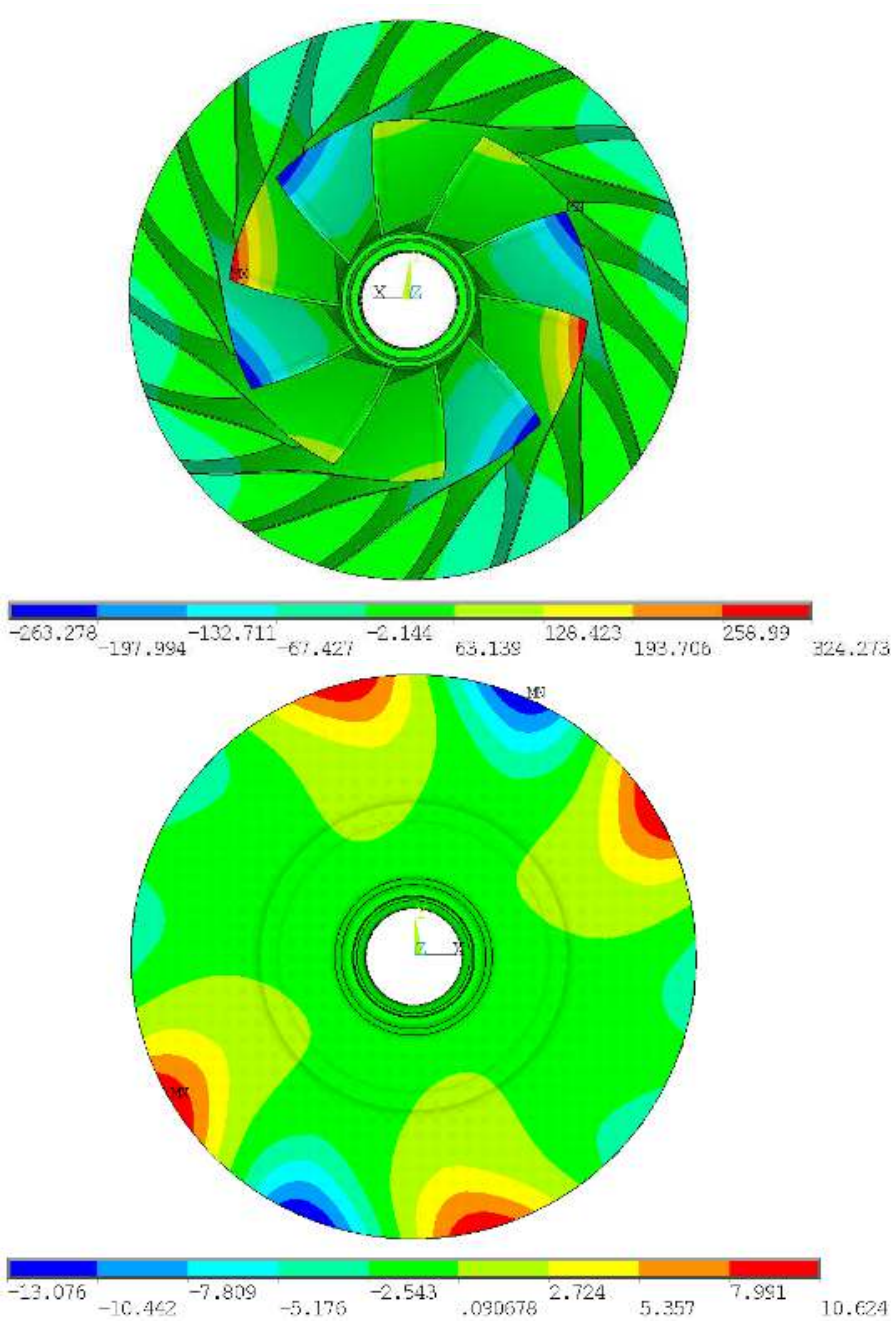

FIGURE 4: Mass normalised FE mode shapes; axial displacement of the 1 st family mode with 4ND. (units : tons $^{-\frac{1}{2}}$ )

quency resolution of the DSP unit. All the tests presented herein were performed with the same excitation system. The measurements have been repeated several times after checking the testing conditions and the results presented in the paper were repeatable.

\subsection{Vacuum chamber}

The impeller with its mounting was installed in a vacuum chamber to perform modal testing. The vacuum chamber was evacuated by a double stage volumetric vacuum pump. The pressure inside the chamber was measured using a Pirani pressure gauge. The vacuum pump was stopped once the desired level of vacuum was reached. The electrical wires necessary for exciting the structure and for measuring input force were passed

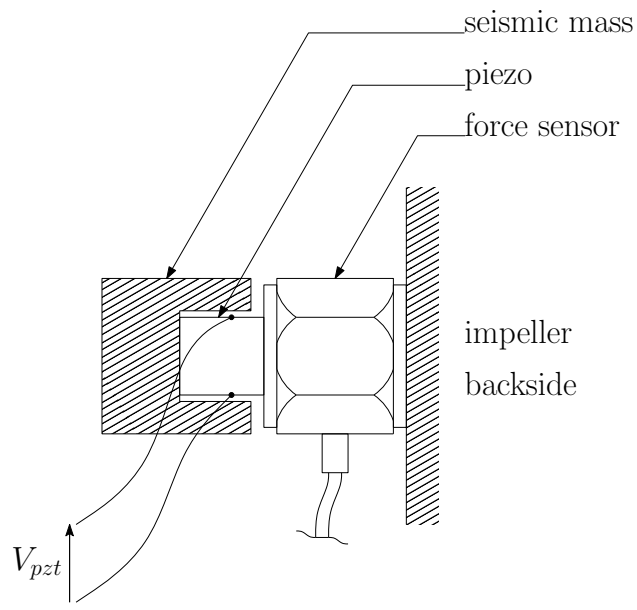

FIGURE 5: Schematic of the piezoelectric shaker

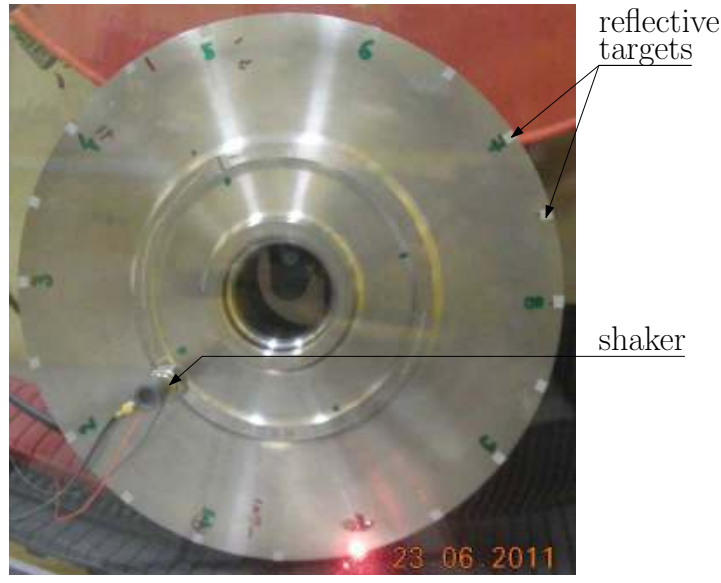

FIGURE 6: Location of excitation device and response measurement points

through the chamber rear wall via a special seal. The front wall was made of transparent PMMA material and designed so that it could withstand external atmospheric pressure and allow the use of an optical measurement system outside the chamber. This feature was also useful for checking that no unwanted contact occurred, for example, between the wires and the blisk, during the measurement. The response of the impeller was measured by a commercial scanning laser vibrometry system (SLDV) [16]. Twenty measurement points were defined on the impeller rear surface close to the trailing edge of the blades (Fig. 6). The response at these locations reached its maximum when the structure vibrated on its modes. Small reflective targets $(4 \times 4 \mathrm{~mm})$ were fixed to improve the light returned back to the vibrometer head and therefore increase the signal to noise ratio. The axis 
of the scanning head was aligned with the axis of the impeller so that the incidence of the laser beam at all the targets was the same. Also, the angle between the laser beam and the normal vector at the targets centre was minimized by operating on the rear side of the impeller. The mass of the reflectors was less than 0.004 grams, which was the same for all the different tests. The evaluated mass-loading effect of these reflectors on natural frequencies was less than $\Delta \omega / 2$. Also, it should be noted that this rig made it easier for the laser beam to reach the response close to the driving point.

\subsection{FRF measurements}

Acquisition and signal generation were carried out by a dualchannel dynamic signal analyzer associated with the SLDV system to measure FRFs relating velocity at each measurement point to the input force at the driving point. A pseudo-random excitation signal was used to avoid the use of time windowing. The FRFs were obtained classically using the $\mathrm{H} 1$ estimator with averaging and overlapping measurement blocks of data at frequency points within a band containing the first flexural mode family of the impeller. For this specific type of structure, it was necessary to use a high frequency resolution $(\Delta \omega)$, because of the high modal density, and to obtain 5 to 10 frequency points within the half power frequency band at resonances so that the damping factor could be identified accurately. This inevitably resulted in relatively long testing times during which it was possible to check that test conditions remained constant (essentially in terms of pressure and temperature).

\subsection{Data reduction}

The FRF data - velocity output at a given point over input force - were analysed by a modal parameters iterative extraction method based on [17]. First, a theoretical FRF form $h_{t h}$ was choosen as the ratio of two polynomials:

$$
h_{t h}(\omega)=\frac{a_{0}+a_{1} \lambda+\ldots+a_{n} \lambda^{n}}{1+b_{1} \lambda+\ldots+b_{n} \lambda^{m}}
$$

where $\lambda=i \omega$, and $a_{j}$ and $b_{j}$ are real unknown coefficients. The orders of the numerator and denominator $(n, m)$ were chosen in accordance with the number of modes responding in the frequency band analysed. The form $h_{t h}(\omega)$ was fitted to frequency response data by minimizing a weighted error function solving for unknowns $a_{j}$ and $b_{j}$

$$
\left(a_{0}, \ldots, a_{n}, b_{1}, \ldots, b_{m}\right)=\operatorname{argmin} \sum_{k=1}^{N_{\omega}} w_{k}\left|h_{t h}\left(\omega_{k}\right)-h_{\text {meas }}\left(\omega_{k}\right)\right|^{2}
$$

where $N_{\omega}$ is the number of measured excitation frequencies in the range of interest and $h_{\text {meas }}$ the FRF data. The algorithm used the damped Gauss-Newton method for iterative search [17]. This solved the direct problem of minimizing the weighted sum of the squared error between the theoretical and the measured frequency response points. The weighting coefficients are user defined : $w_{k}=\left|h_{\text {meas }}\left(\omega_{k}\right)\right|^{2}$. Once the polynomial coefficients had been found, the residues and the poles of the partial fraction expansion of the ratio of the two polynomials were obtained numerically. Real and imaginary parts of identified poles were derived afterward to extract natural frequencies and damping ratios as partial fraction expansion could be identified with the classical expression of $h_{t h}$ in term of modal parameters:

$$
h_{t h}(\omega)=\sum_{k=1}^{10} \frac{A_{k}}{\lambda-\lambda_{k}}+\frac{\bar{A}_{k}}{\lambda-\bar{\lambda}_{k}}+U+\frac{V}{\omega^{2}}
$$

where $\lambda_{k}=-\zeta \omega_{k}+i \omega_{k} \sqrt{\left(1-\zeta^{2}\right)}$ is the $k^{\text {th }}$ pole and $\bar{\lambda}_{k}$ its complex conjugate and $A_{k}$ is the corresponding complex modal residual and $\bar{A}_{k}$ its conjugate. Coefficients $U$ and $V$ aim to take into account the influence of out of range modes. The modal residues were used to identify mode shapes and their nodal diameter numbers $(N D)$. As shown later, the use of vacuum conditions allows very good direct identification of the number of peaks. In fact, the expected total number of ten peaks for the impeller, which had ten sectors, was clearly seen on the FRFs. Expected doubles modes (pairs) with 1,2,3 and 4 NDs where found at lightly split frequencies. Both modes within a pair were excited by the single point excitation location chosen. It should also be mentioned that numerical analysis showed that the first mode family was also well separated from the others.

The measured data were therefore reduced to ten damping ratios, natural frequencies and complex residues. An example of measured FRF data (displacement over force) superimposed with the FRF synthesized as the sum of the modal participations is shown in Fig. 7

\section{RESULTS AND DISCUSSION \\ 3.1 Frequencies and damping ratios}

The identified natural frequencies and damping ratios are gathered in table 1. These reduced data are sorted by ascending frequency and normalized. For each frequency, the $N D$ of the corresponding mode shape is given. The relative distance in frequency within a pair of the same $N D$ ranges from $0.06 \%$ to $0.1 \%$ depending on the ND. It is worth mentionning that damping ratios at ambient pressure are more than 30 times higher than at 10mbars. Uncertainties on modal parameters values have been evaluated by analyzing each one of the 20 measured FRFs separately and then by looking at the scattering of the results. Theoretically, these values should be the same. Although, possible errors in measurements or in identification process had lead 
10 mbars, ch. 9
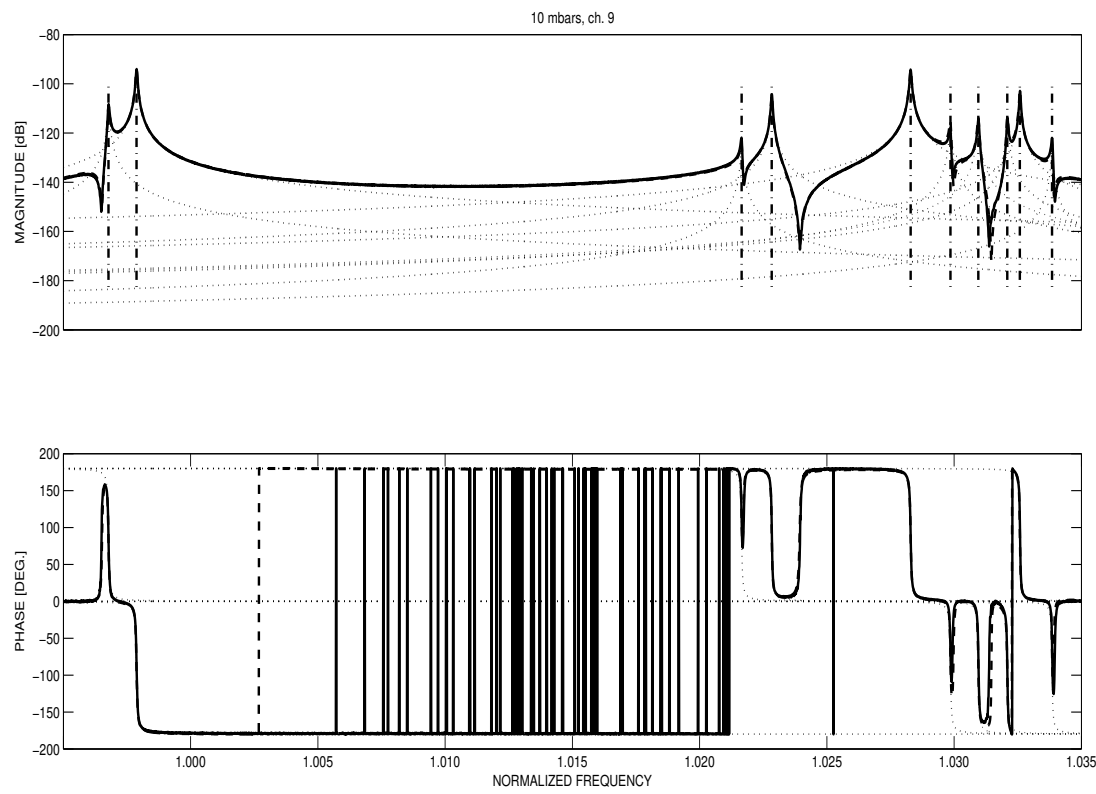

FIGURE 7: Typical FRF: measured FRF (dashed bold curve), synthesized FRF (solid bold curve), modal participation of each mode (thin curves)

to slightly different values. The mean over the 20 individual results obtained is used as an estimate of the true value for a modal parameter and these are reported in table 1 . The amplitude of the scattering around mean values is represented on diagrams in Fig. 9 by vertical bars. The height of a vertical bar is equal to the difference between the highest and the smallest of the different identified values for a particular modal parameter. Several remarks can be made looking at these diagrams. First, the natural frequencies are very accurately estimated as the amplitude of the scattering is limited to $\pm 0.001 \%$, which is on the order the frequency resolution of the DSP unit $\Delta \omega$ (bars height have been magnified 1000 times on the diagram for readability). Despite the uncertainty on damping ratios, their extremely relative low values are confirmed. The relative error on damping ratios at $100 \mathrm{mbar}$ are on the order of $\pm 20 \%$. Note that certain modes - $0 N D, 1 N D b, 2 N D b, 3 N D b, 4 N D a, 5 N D$ - are estimated with more accuracy. As revealed by the modal extraction, these latter have relatively more important modal participations. This can be explained by the circumferantial of the shaker w.r.t. nodal diameters which makes some mode globally predominant in the set of FRFs (see a sample in Fig. 7). This could be optimized $a$ posteriori concerning modes with $1,2,3,4 N D s$. Note that modes with $0 N D$ and $5 N D$ are accurately estimated. Regarding the spread of damping ratios within a pair of modes, note that it is in the range of uncertainty. Interestingly, it can be mentioned from Fig. 9 that damping ratios for mode with $N D$ numbers $1,3,5$ are lower than the others (with $N D=0,2,4$ ).

In the case where the pressure was 1 bar, the FRFs could not be fitted satisfactorily through all the frequency range. The damping ratios estimated at $1 \mathrm{~atm}$ scattered between 0.8 and 1.2. The results in terms of modal parameters are given for all the pressures tested for the first 2 modes only. Due to the difficulty of extracting modal parameters from the tests at $1 \mathrm{bar}$, a direct comparison of the FRFs is proposed (Fig. 8).

The influence of air on frequencies is quite mild as frequency shifts between 10 mbar and 1000 mbar does not exceed $0.3 \%$, validating the first order perturbation approach on which the principle of vacuum vibratory measurements is based. Such low variations justify a posteriori the care taken over the experimental setup. Nevertheless, due to the low number of pressure points available, it seems risky to assess the linearity with respect to pressure without extra measurements. Additional tests would be especially beneficial when starting at a high pressure limit of less than 1 bar and chosen so that identification is easy or at least feasible. In case an attempt of linear fitting were made, one could notice the tendency of points corresponding to damping measured at $10 \mathrm{mbar}$ to be below the line joining the points of measurements at 100 or 1000 mbar (Fig 10). Such deviation from a linear behaviour, if confirmed, could be compared to an analogous behaviour noticeable on similar measurements performed on a fan blade and reported in [5]. This would weaken the hypothesis of linear acoustic behaviour. Another remark is 


\begin{tabular}{|c||r|r||r|r||r|r|}
\hline \multicolumn{1}{|c||}{} & \multicolumn{2}{c||}{$\mathrm{P}=10$ mbar } & \multicolumn{2}{c||}{$\mathrm{P}=100$ mbar } & \multicolumn{2}{c|}{$\mathrm{P}=1$ bar } \\
\hline$N D$ & $f_{\text {norm }}$ & $\zeta_{\text {norm }}$ & $f_{\text {norm }}$ & $\zeta_{\text {norm }}$ & $f_{\text {norm }}$ & $\zeta_{\text {norm }}$ \\
\hline $2 \mathrm{a}$ & 1.00175 & 0.029 & 1.00145 & 0.20 & 0.99865 & 1.0 \\
$2 \mathrm{~b}$ & 1.00285 & 0.029 & 1.00260 & 0.18 & 1.00055 & 0.7 \\
$3 \mathrm{a}$ & 1.02665 & 0.023 & 1.02630 & 0.16 & $\mathrm{ni}$ & $\mathrm{ni}$ \\
$3 \mathrm{~b}$ & 1.02785 & 0.025 & 1.02735 & 0.20 & $\mathrm{ni}$ & $\mathrm{ni}$ \\
0 & 1.03330 & 0.030 & 1.03295 & 0.13 & $\mathrm{ni}$ & $\mathrm{ni}$ \\
$\mathrm{4a}$ & 1.03485 & 0.026 & 1.03440 & 0.12 & $\mathrm{ni}$ & $\mathrm{ni}$ \\
$4 \mathrm{~b}$ & 1.03595 & 0.025 & 1.03540 & 0.11 & $\mathrm{ni}$ & $\mathrm{ni}$ \\
$\mathrm{1a}$ & 1.03710 & 0.023 & 1.03670 & 0.10 & $\mathrm{ni}$ & $\mathrm{ni}$ \\
$1 \mathrm{~b}$ & 1.03760 & 0.022 & 1.03730 & 0.10 & $\mathrm{ni}$ & $\mathrm{ni}$ \\
5 & 1.03885 & 0.024 & 1.03820 & 0.11 & $\mathrm{ni}$ & $\mathrm{ni}$ \\
\hline
\end{tabular}

TABLE 1: Evolution of normalized modal frequencies $f_{\text {norm }}$ and normalized critical damping ratios $\zeta_{\text {norm }}$ according to ambient pressure P. When double modes exist, index $a$ or $b$ is used to distinguish them. "ni" stands for "not identified"
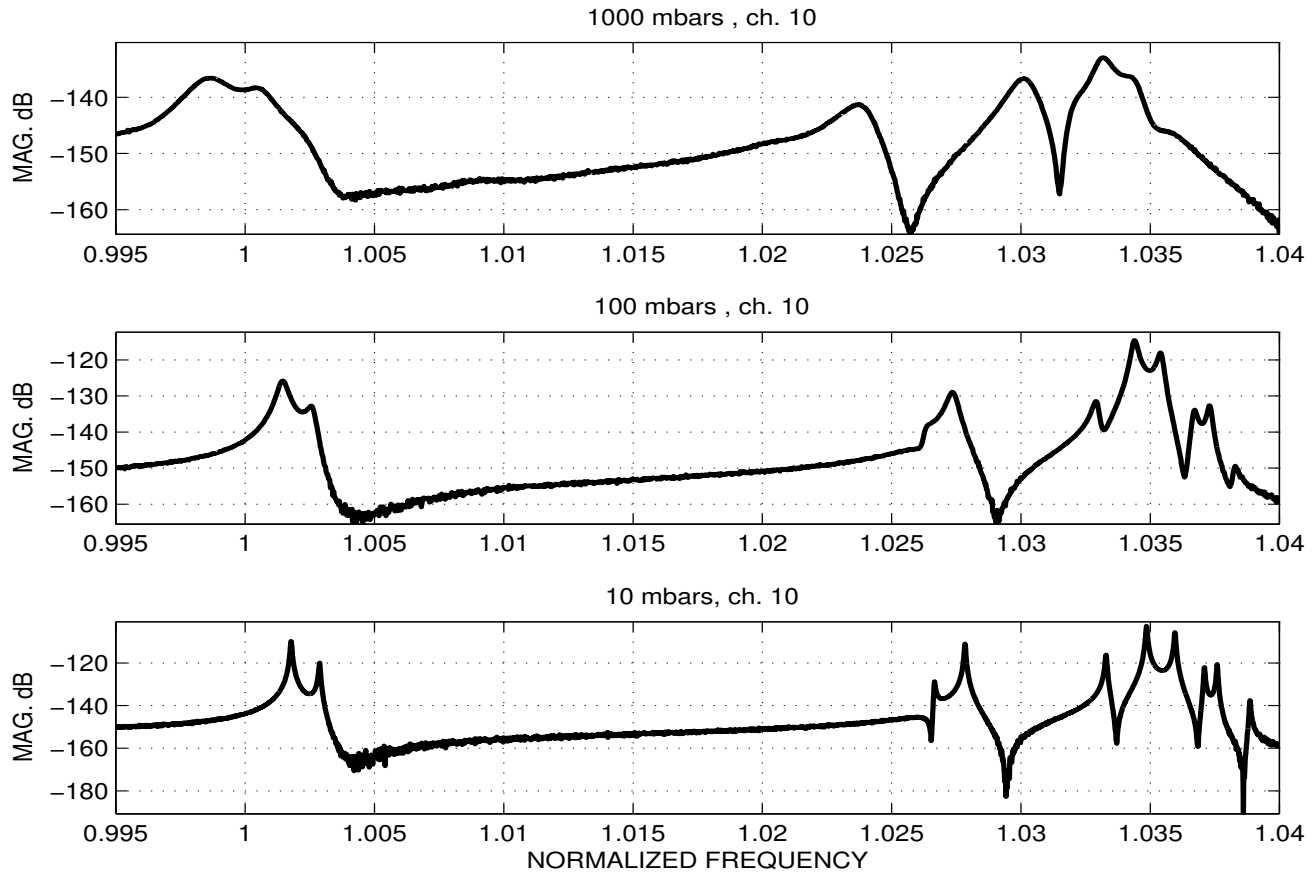

FIGURE 8: Direct comparison of FRFs measured at point 10 at 10,100 and 1000 mbars

that linearly fitting only through points located above a certain limit of pressure - 100 mbar for example - would result here in overestimating material damping. The influence of the choice of measurement points included in the linear fitting is illustrated in table 2, with different estimates of normalized critical material damping ratios $\zeta_{0 \text { norm }}$ in vacuum. Considering the lack of knowledge on what the behaviour of the structure is under $10 \mathrm{mbar}$, and the difficulty of performing a substantiated linear fitting, it seems reasonable to conclude that the value of damping measured at 10 mbar is a good approximate of material damping. 


\begin{tabular}{|c||c|c|c|}
\hline$N D$ & $\zeta_{\text {Onorm }}(10-100 \mathrm{mbars})$ & $\zeta_{\text {Onorm }}(100-1000 \mathrm{mbars})$ & $\zeta_{\text {Onorm }}(10-100-1000 \mathrm{mbars})$ \\
\hline 2a & 0.010 & 0.107 & 0.058 \\
$2 \mathrm{~b}$ & 0.013 & 0.117 & 0.065 \\
\hline
\end{tabular}

TABLE 2: Interpolated normalized critical material damping ratios $\zeta_{\text {Onorm }}$ in vacuum, based on the measurements performed at the indicated pressure levels
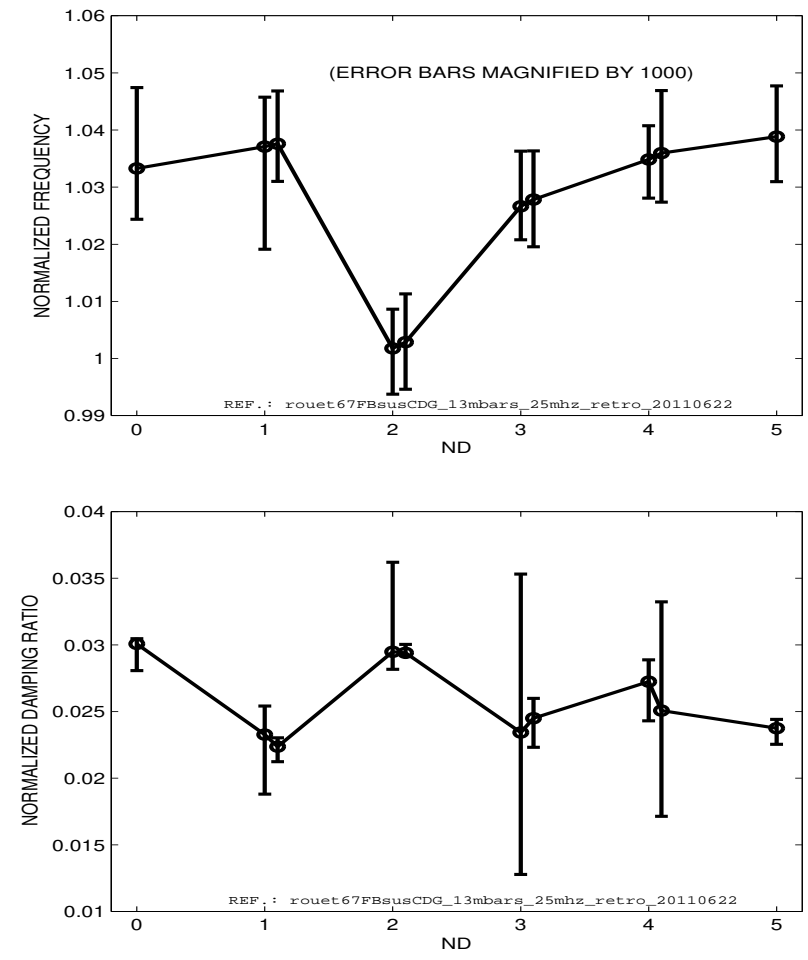

FIGURE 9: Damping ratios and natural frequencies over NDs at $10 \mathrm{mbars}$

\subsection{Comparison with other available data}

In spite of confidentiality reasons that prevent us from publishing raw data, it seems important to place the present results in relation to other available data [9, 18]. The purpose is to provide tendencies of the conditions under which air is likely to have a significant impact on damping measurements, and thus indicate when experiment designers should be concerned about it.

A simple model taken from the literature [19] was used to give an analytical framework. Let us consider the case of a plate oscillating harmonically. Its behavior is assumed to be similar to that of an impeller main blade. When replacing the plate surface by a distribution of harmonically oscillating sources, the air loading effect has different simplified expressions according to
Norm. damping ratio

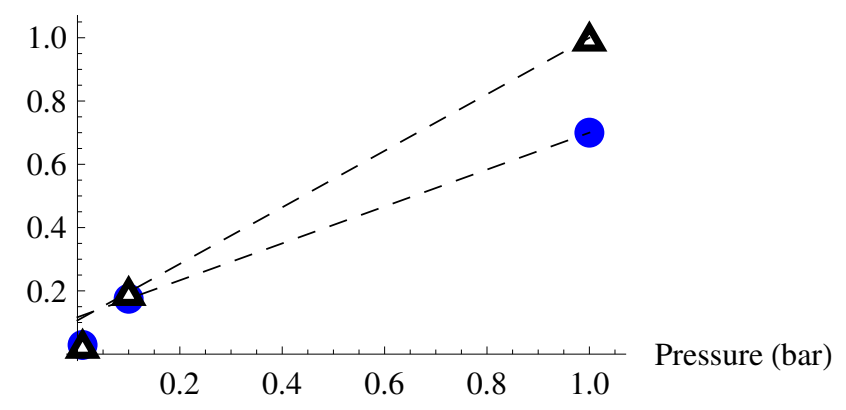

FIGURE 10: Normalized critical material damping ratios vs pressure level for mode $2 \mathrm{a}$ (triangles) and mode $2 \mathrm{~b}$ (circles)

the value of parameter $k=\frac{\omega a}{2 c}$, where $a$ is a characteristic length of the structure, $\omega$ the excitation frequency and $c$ the acoustic wave speed in surrounding air. The distinction between low frequencies and high frequencies can be made when $k<1$ and when $k>3$, respectively. The intermediate frequency range represents a transition between both types of loadings.

An analogy is performed between the dynamical behaviours of a main blade - vibrating on its first flexural mode - and of a rectangular simply supported plate - also vibrating on its first flexural mode of natural frequency $\omega_{1}$. In spite of the fact that geometry and boundary conditions are different, the tendencies can be expected to be similar regarding the material parameters. This helps when comparing the aerodynamic dampings obtained between similar impellers made of different materials.

Let $a, b$ and $h$ be the plate length, width and thickness, $\rho_{m}$ the density of the plate material. $a$ and $b$ are chosen as equal to the average span and chord of the impeller main blade studied, and $\omega_{1}$ is equal to the average frequency of the first flexural mode family. The case studied corresponds to a low frequency coupling because $k<1$. The critical damping ratio associated with aerodynamical damping is the ratio between terms $C_{a}$ (of Eq11) and $C_{c r}=2 \omega_{1} M$ [19]:

$$
\frac{C_{a}}{C_{c r}}=0.156\left(\frac{b}{a}+\frac{a}{b}\right) \frac{\rho}{\rho_{m}} \frac{c_{m}}{c}
$$


This equation is obtained for Poisson coefficient $v=0.3$. $c_{m}=\sqrt{\frac{E}{\rho_{m}}}$ is the speed of sound in the material with Young modulus $E$ and material density $\rho_{m}$. If the temperature is constant, then $c$ does not depend on $P$ and $\rho$ is proportional to $P$. Thus, for a given air pressure, air damping depends on the plate geometry and material, and on modal parameters (natural frequency and mode shape, since the pressure field depends on the latter).

Eq77 provides clues to predict the impact of air on total damping. For example, air damping should not depend on the absolute dimensions of the plate but only on its aspect ratio $\frac{a}{b}$. Another tendency is that increasing $\rho_{m}$ should result in decreasing $c_{m}$ and then lowering air damping, as expected. It is also noticeable that blade thickness does not seem to play a role in air damping. But this would be different for higher values of coefficient $k$.

At this stage, a comparison between the experimental results from the previous paragraph and those provided by [18] can be proposed. The characteristic dimensions are different, Kammerer's impeller is larger $(400 \mathrm{~mm}$ diameter compared to $260 \mathrm{~mm}$ ) but with a similar blade aspect ratio. It is made of aluminum instead of titanium. It has 7 main blades instead of 10 and they seem to be thicker. In terms of modal properties, the frequencies of the first mode family modes of the present impeller are located between the frequencies of the first and second family modes of Kammerer's $(1350 \mathrm{~Hz}, 2875 \mathrm{~Hz})$. The first family modes have similar mode shapes on both structures. The comparison is performed using data extrapolated from Kammerer's mode 1 (assumed identified on an isolated blade). Assuming that aerodynamical damping on impellers also depends on aspect ratios, Eq 7 allows predicting that the ratio between the air damping provided to Kammerer's impeller and air damping on the present one should be equal to 1.7. Averaging the air damping ratios identified herein on all the modes of the family provides a result in line with the tendency expected, with higher values on the aluminum impeller than on the titanium one. The discrepancy is about $60 \%$ at $P=1$ bar and about $20 \%$ at $P=100$ mbar. Therefore, in spite of the great simplicity of the analytical model used to correlate data obtained from quite different impellers, the real air damping ratio values measured here are in agreement with those measured and reported by other authors.

Concerning material damping, it can be concluded from the experiments described herein that the present material damping values are significantly lower than those given in [18] but of the same order of magnitude as the lowest values available in the literature [5]. This can be explained by the fact that our impeller was tested with practically free boundary conditions and with contactless instrumentation rather than with clamping and more intrusive instrumentation, possibly leading to additional damp- ing. But the most important conclusion is that for such a structure the presence of air is highly likely to pollute material damping measurement as its relative contribution to total damping is much greater than previously reported. This makes using vacuum measurements essential.

\section{CONCLUSIONS AND PROSPECTS}

In this paper, a dedicated experimental setup was proposed to identify the dynamical characteristics of a radial impeller in terms of natural frequencies and nodal diameter mode shapes, and to assess material damping by trying to minimize different spurious effects. Although blisk structures are known to be very lightly damped, it has been noted that the direct location of resonances on measured FRFs was not obvious when modal tests on the impeller were conducted under ambiant pressure. The resonances seemed heavily damped and the high modal density was probably an additional difficulty for the analysis. Nonetheless, the blisk was tested carefully under practically free-free boundary conditions and its response measured by SLDV.

The decision to operate inside an evacuated chamber to improve testing conditions highlighted the remarkable property of vacuum. Then, all the expected resonance peaks in the frequency range could be clearly located on the FRF data, revealing modes with 0 to 5 nodal diameters with split pairs ( 1 to 4 ND). The analysis of data confirmed a strong dependency of damping ratios on pressure on the first flexural mode, as the ratio of damping in air and at vacuum $\zeta / \zeta_{0}$ was on the order of 30 . Although, some published experimental works report a non linear evolution of damping w.r.t. pressure when pressure is close to zero, the principle of vibratory measurements in vacuum based on the linearity of modal parameter evolution as a function of pressure has been used to assess material modal damping, showing the limits of the method. Additional pressure points would be necessary to fit a nonlinear relationship - which remains to be determined - and to obtain more accurate material damping measurments.

A simple analytical model was used in order to show the influence of the different physical parameters involved in the damping ratio of a vibrating flat plate in surrounding air: aspect ratio, material density, frequency and mode shape, air density. Assuming that the analytical model was applicable, a quantitative comparison has been performed with results from a similar structure found in the literature. Considering the first flexural mode shapes, the ratio of damping in air and at vacuum was lesser $\left(\zeta / \zeta_{0}=8\right)$ in these latter results but the analytical model gave a way to correlate satisfactorily both evaluations of air damping. Given the physical parameters of the two impellers, the simple model predicted that damping in air of the aluminium impeller was 1.7 times higher. Although, the fact that our damping ratios at vacuum are very much lower makes the ratios $\zeta / \zeta_{0}$ of the two studies consistant. Some differences from our case should be taken into account: especially, the blisk was 
clamped on a shaft made of different material, it instrumentation was probably more intrusive and aluminium may have more material damping than titanium.

Further measurements would focus on other mode families to perform analogous analysis with higher modes. The first two mode families for a similar structure have been tested in reference [9]. It was found that the influence of surrounding air on the second family was two to three times lower w.r.t. the first one. Although our impeller geometry and material are different it seems that we could expect the same behavior.

The role of rotation also deserves re-examination. Indeed, in [5], the authors concluded from rotating tests performed on a single plate-like blade that rotation effects were not significant, but suspected more complex material damping processes for wide chord and three-dimensional slender blisks. Specific experiments such as in [20] could be performed.

\section{ACKNOWLEDGMENT}

This work was partially funded by the French National Research Agency (ANR) in the framework of its Technological Research COSINUS program (IRINA project, ref. ANR 09 COSI 00801 IRINA).

\section{References}

[1] Blevins, R. D., 2001. Flow induced vibration. Krieger Publishing Company, Malabar, Florida.

[2] Laxalde, D., Gibert, C., and Thouverez, F., 2008. "Experimental and numerical investigations of friction rings damping of blisks". In Proceedings of ASME Turbo Expo, 2008.

[3] Beards, C. F., 1996. Structural vibration: analysis and damping. Elsevier.

[4] Harris, C. M., and Piersol, A. G., 2002. Harris' Shock and Vibration Handbook. McGraw-Hill.

[5] Srinivasan, A. V., Cutts, D. G., and Sridhar, S., 1981. Turbojet engine blade damping. Tech. Rep. NASA-CR165406, NASA.

[6] Kielb, J. J., and Abhari, R. S., 2003. "Experimental study of aerodynamic and structural damping in a full-scale rotating turbine". Journal of Engineering for Gas Turbines and Power - Transactions of the ASME, 125(1), pp. 102-112.

[7] Jeffers, T. R., Kielb, J. J., and Abhari, R. S., 2000. "A novel technique for the measurement of blade damping using piezoelectric actuators". In Proceedings of ASME Turbo Expo, 2000.

[8] Kammerer, A., and Abhari, R. S., 2008. "Experimental study on impeller blade vibration during resonance - Part 1: blade vibration due to inlet flow distortion". In Proceedings of ASME Turbo Expo, 2008.
[9] Kammerer, A., and Abhari, R. S., 2008. "Experimental study on impeller blade vibration during resonance - Part 2: blade damping". In Proceedings of ASME Turbo Expo, 2008.

[10] Zemp, A., Ribi, B., and Abhari, R. S., 2011. "Experimental investigation of forced response impeller blade vibration in a centrifugal compressor with variable inlet guide vanes Part 1: blade damping". In Proceedings of ASME Turbo Expo, 2011.

[11] Lesueur, C., 1988. Rayonnement acoustique des structures (Acoustic Radiation of Structures, in french). Eyrolles.

[12] David, B., 1999. "Caractérisation acoustique des structures vibrantes (Acoustic Characterization of Vibrating Structures, in french)". $\mathrm{PhD}$ thesis, University Paris 6.

[13] David, B., 1995. "Using vacuum to measure the acoustical efficiency". In International Symposium on Musical Acoustics ISMA'95: July 2-5, 1995, Dourdan, France. In S.F.A. (Ed.), pp. 379-385.

[14] http://www.physikinstrumente.com/pztactuators/1_6t.html, 2011.

[15] Szwedowicz, J., Gibert, C., Sommer, T. P., and Kellerer, R., 2006. "Numerical and experimental damping assessment of a thin- walled friction damper in the rotating set-up with high pressure turbine blades ". In Proceedings of ASME Turbo Expo, 2006.

[16] http://www.polytec.de/us/products/vibrationsensors/scanning-vibrometers/psv-400-scanning vibrometer, 2011.

[17] Dennis, J. E. J., and Schnabel, R. B., 1983. Numerical Methods for Unconstrained Optimization and Nonlinear Equations. Englewood Cliffs, NJ: Prentice-Hall.

[18] Kammerer, A., 2009. "Experimental Research into Resonant Vibration of Centrifugal Compressor Blades". PhD thesis, ETH Zurich.

[19] Harris, C. M., and Crede, C. E., 1961. Shock and Vibration Handbook. McGraw-Hill.

[20] Jean, P., Gibert, C., Dupont, C., and Lombard, J. P., 2008. "Test-model correlation of dry-friction damping phenomena in aero-engines". In Proceedings of ASME Turbo Expo, 2008. 